\section{Prevalence of dermatofibrosarcoma protuberans in Saudi Arabia over 24 years. A retrospective single-institution study}

\author{
Najla A. Al-Dawsari, MD, FAAD, \\ Salwa S. Al Sheikh, MD, FCAP.
}

\begin{abstract}
Objectives: To conduct a descriptive clinicopathological and demographic analysis of dermatofibrosarcoma protuberans among Saudi patients attending a tertiary care center in the eastern province of Saudi Arabia.
\end{abstract}

Methods: This retrospective, single-center study reviewed the medical records and pathology reports of Saudi patients attending a tertiary center (Johns Hopkins Aramco Health Care) in Dhahran, Saudi Arabia from 1995 to 2019 to identify all cases of dermatofibrosarcoma protuberans. Demographic and phenotypic data were also analyzed.

Results: Thirty-five Saudi patients were identified as having dermatofibrosarcoma protuberans. Females constituted $68.6 \%$ of patients. The trunk was the most common site of involvement (54.3\%), followed by the lower extremities (34.3\%), upper extremities $(8.6 \%)$, and head and neck $(2.9 \%)$. The mean tumor size was $2.9 \mathrm{~cm}$ (standard deviation: \pm 2.0 ). There was only one case of distant metastasis of fibrosarcomatous dermatofibrosarcoma protuberans.

Conclusions: Dermatofibrosarcoma protuberans was not frequently encountered in Saudi patients in a tertiary center that treats approximately 187,668 patients. Dermatofibrosarcoma protuberans disease patterns and demographic data were similar to those reported worldwide. Further studies are required to characterize dermatofibrosarcoma protuberans in the Saudi population.

Keywords: skin cancer, sarcoma, Saudi Arabia

Saudi Med J 2021; Vol. 42 (12): 1362-1365 doi: 10.15537/smj.2021.42.12.20210440

$\mathrm{D}$ ermatofibrosarcoma protuberans (DFSP) is categorized as a "fibroblastic/myofibroblastic tumor of intermediate malignancy (rarely metastasizing)" in the classification of soft tissue tumors by the World Health Organization in 2013. ${ }^{1}$ The annual incidence rate of DFSP is $0.8-4.1$ cases per million persons. ${ }^{2,3}$ It is a superficial mesenchymal neoplasm of fibroblastic origin. Dermatofibrosarcoma protuberans usually develops at 30-40 years and often follows a long course of more than 10-15 years. Dermatofibrosarcoma protuberans lesions have been described in children and are uncommon in the elderly, and congenital lesions have also been reported. The female or male predominance of this tumor remains controversial. The tumor is slow-growing and has a high tendency for local recurrence (20-50\%); however, it rarely metastasizes to other sites in the body via the lymph or blood. Dermatofibrosarcoma protuberans is an aggressive tumor with a tendency for local invasion and recurrence, particularly in the head and neck region (50-75\%). ${ }^{4,5}$ Histologically, this neoplasm shows a distinctive, dense spindle cell proliferation of uniform bland-looking cells that are arranged in a storiform or cartwheel-like growth pattern in the dermis, which also shows diffuse infiltration of subcutaneous fat. Immunohistochemistry shows that CD34 is the hallmark of DFSP, with P53 protein positivity observed during fibrosarcomatous transformation. In most cases, cytogenetic studies can identify a supernumerary ring chromosome. The $\mathrm{t}(17 ; 22)$ (q22; q13) gene undergoes translation, which leads to the fusion of the COL1A1 and PDGFB genes.

Virtually all DFSP cases contain fusion genes, with COL1A1-PDGFB being the most common. Less common fusions include COL1A2-PDGFB, COL6A3PDGFD, and EMILIN2-PDGFD. ${ }^{6,7}$

After reviewing the literature on DFSP from several countries, we concluded that there is a lack of articles describing the epidemiological data and clinical characteristics of DFSP in Saudi Arabia. To address this knowledge gap, we aimed to describe the clinical and histological characteristics of DFSP and investigate its prevalence among Saudi patients.

Methods. This record-based single-center retrospective study included surgical pathology reports from January 1995 to December 2019 of cases with a confirmed diagnosis of DFSP at Johns Hopkins Aramco Healthcare, Dhahran, Saudi Arabia. We applied a combination of free text search and institutional code for malignancy. The Institutional Review Board of the medical center, from reviewed and approved our study

Disclosure. Authors have no conflict of interests, and the work was not supported or funded by any drug company. 
protocol. The study design followed the principles of the Declaration of Helsinki. The requirement for informed consent was waived by the Institutional Review Board. Data were collected from a tertiary care center in the eastern province of Saudi Arabia that exclusively treats Saudi Aramco's employees and families along with the hospital's employees and their families. The children of employees are not eligible for treatment if their parents retire, after age the age of 20 years for the male dependents, or after marriage for female dependents. Some executive employees maintain their eligibility even after retirement. All patients with an eligible treatment status were reviewed and non-Saudi patients were excluded. Data were collected from the medical records of 35 Saudi patients diagnosed with DFSP. Reports were manually reviewed, and the following parameters were extracted: date of biopsy and re-excision, medical record number, age, gender, nationality, pathological diagnosis, histopathological stains, anatomic location, and preoperative lesion size. Medical records were reviewed to obtain the preoperative diagnosis, treatment plan, adjunctive chemotherapy or radiation therapy, and evidence of recurrence or metastasis. The survival rate was obtained by the national identification numbers of patients, through communication between the hospital admission office and Saudi Civil Affairs.

Statistical analysis. A computerized search was exported to Microsoft Excel and used for the analysis. Data entry and analysis were performed using SPSS for Windows, version 24.0 (IBM Corp., Armonk, NY, USA). Categorical data are presented as frequencies and percentages, and numerical data are presented as mean $\pm \mathrm{SD}$.

Results. During the 24-year period, the total population treated at the institute was 187,668 . Of these, 35 Saudi patients were diagnosed with DFSP. The mean age of patients diagnosed with DFSP was 37.9 years SD $( \pm 15.4)$ with females comprising $68.6 \%$ of the patients. The demographic and clinical characteristics of the patients are summarized in Table 1.

Twenty-nine lesions had the typical histopathological pattern of DFSP. Three lesions showed giant cell fibroblastoma transformation, one lesion was a pigmented variation of DFSP, one had extensive myxoid changes, and one had fibrosarcomatous changes. Over the years, multiple histopathological stains have been used. Twenty-one patients had CD34 positive tumors while 13 tumors were $S 100$ positive. Seven tumors were negative for vimentin and actin, 2 tumors were desmin-negative, one tumor was CD117 negative, 2 tumors were CD68 positive, and 2 tumors were CD68 negative. All patients underwent wide local excision
Table 1 - Characteristics of dermatofibrosarcoma protuberans among Saudis at a tertiary care center in eastern Saudi Arabia.

\begin{tabular}{lcccc}
\hline Characteristics & \multicolumn{2}{c}{ Gender } & Chi-square & $P$-value \\
& Male & Female & & \\
\hline Location & & & & \\
Head and neck & 0 & $1(100)$ & 3.66 & 0.30 \\
Lower extremity & $2(16.7)$ & $10(83.3)$ & & \\
Trunk & $7(36.8)$ & $12(63.2)$ & & \\
Upper extremity & $2(66.7)$ & $1(33.3)$ & & \\
Pre-operative size & & & & \\
$<2.00$ cm & $4(26.7)$ & $11(73.3)$ & 1.58 & 0.45 \\
$2.00-3.00$ cm & $2(28.6)$ & $5(71.4)$ & & \\
$>3.00$ cm & $5(50.0)$ & $5(50.0)$ & & \\
Age at diagnosis (years) & & & \\
Less than 18 & $2(40.0)$ & $3(60.0)$ & 0.22 & \\
$19-40$ & $5(31.3)$ & $11(68.8)$ & & \\
41 and above & $4(28.6)$ & $10(71.4)$ & & \\
\hline
\end{tabular}

with tumor-free margins ( 2 patients received treatment at another center). Thirty patients $(85.7 \%)$ had clear histopathological margins after the first excision. One patient underwent re-excision because of positive resection margins. One tumor was less than $0.1 \mathrm{~cm}$ of the lateral margin. We were unable to find excision details in 3 patients. One patient received radiation after re-excision.

Twelve patients had no recurrence for up to 5 years, while 4 patients had no recurrence for 6 to years and 6 patients had no recurrence for more than 11 years. Recurrence records were not available for 12 patients. Twenty one of 35 patients had no metastasis while records on metastasis were not available for 13 patients. There was one case of fibrosarcomatous DFSP in a 37-year-old man who exhibited lung metastasis without local recurrence less than a year after his tumor had been excised with clear margins, which was treated with imatinib mesylate prior to metastasis resection for 3 months; the patient then underwent lobectomy of the left lower lung. Figure 1 depicts a chest computed tomography scan of the metastatic lesion. All 35 patients diagnosed since 1995 were alive as of February 2021.

Discussion. According to a Saudi report on cancer incidence in 2015, skin cancer (excluding lip and melanoma) ranks 44th among all cancers. It is unclear whether this report classified DFSP as skin cancer or soft tissue sarcoma, which is the 47 th most common cancer among the Saudi population. ${ }^{8}$ In a retrospective 
study performed at our center between 1994 and 2014, we observed that DFSP was the sixth most common cutaneous malignancy among Saudi patients; basal cell carcinoma was the most common, followed by squamous cell carcinoma, mycosis fungoides, malignant melanoma, and Kaposi's sarcoma. ' Several studies on skin cancer conducted in different regions of Saudi Arabia have mentioned DFSP; these data are summarized in Table 2.9-14

The clinico-demographic data of DFSP among Saudis have not been specifically discussed in the literature. Kreicher et $\mathrm{al}^{3}$ studied the largest cohort of
7,250 DFSP cases in the United States between 2000 and 2010 . The overall incidence was $4.1 /$ million personyears. The incidence among women was 1.14 times higher than that among men, and the most susceptible age group was 20-39 (42\%) years. The trunk was the most common anatomical site, except in elderly men. Of all cases, $41.7 \%$ presented on the trunk, $21.2 \%$ on the upper limb, $20.8 \%$ on the lower limb, and $12.9 \%$ on the head. The 10-year relative survival rate was $99.1 \%$.

Study limitations. This study is a retrospective design and lack of specific details on the precise size of the resected margins. We also could not follow-up all our

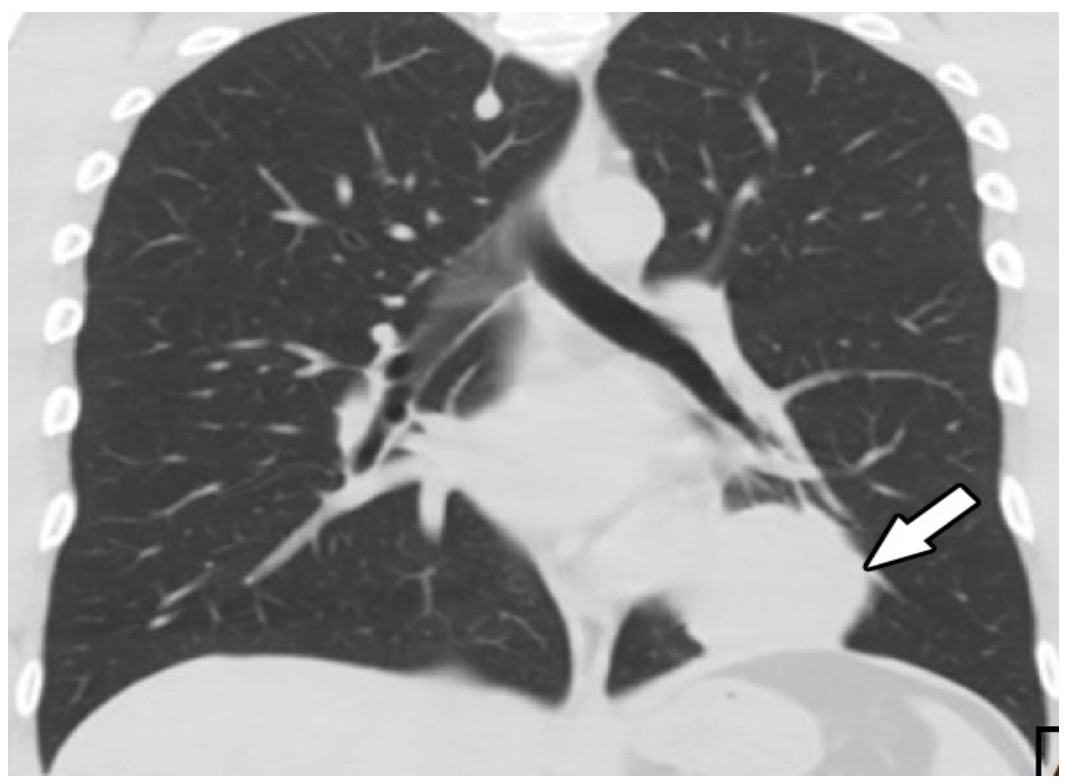

Figure 1 - PChest computed tomography showing a soft tissue mass $(5 \times 3.5 \times 2.4 \mathrm{~cm})$ that is attached to from the left side of the pericardium/left hemidiaphragm on the left lower lobe of the lung.

Table 2 - Published data on DFSP in several regions in Saudi Arabia.

\begin{tabular}{|c|c|c|c|c|c|c|}
\hline Authors & $\begin{array}{l}\text { Number of cases per } \\
\text { total skin cancer cases }\end{array}$ & $\begin{array}{l}\text { Mean age of } \\
\text { DFSP patients }\end{array}$ & $\begin{array}{l}\text { Male/ } \\
\text { Female }\end{array}$ & Region & Study duration & Study population \\
\hline Al-Maghrabi et al ${ }^{10}$ & $3 / 107$ & Not recorded & $1 / 2$ & Baha & $1990-2003$ & Not specified \\
\hline Alwunais et $\mathrm{al}^{14}$ & $5 / 27$ & Not recorded & & Dammam & 2008-2014 & Not specified \\
\hline Hafez et $\mathrm{al}^{13}$ & $1 / 100$ & Not recorded & $1 / 0$ & Southwest & $2012-2016$ & $\begin{array}{l}\text { Saudi (94\%) } \\
\text { and Non-Saudi }\end{array}$ \\
\hline Mufti $^{12}$ & $6 / 106$ & 40.6 & $2 / 4$ & West & 2000-2010 & Saudi \\
\hline Albasri et al ${ }^{11}$ & $\begin{array}{c}12 / 202 \\
\text { Chest }\end{array}$ & 32.2 & $8 / 4$ & $\begin{array}{l}\text { Al-Madina } \\
\text { Al-Munawara }\end{array}$ & 2006-2017 & Saudi \\
\hline Al-Dawsari et $\mathrm{al}^{9}$ & $11 / 204$ & 40 & $5 / 6$ & Dhahran & 1994-2014 & Saudi \\
\hline
\end{tabular}


patients for prolonged periods because our institution only found certain patients eligible for treatment. The study is a descriptive in a closed healthcare system and cannot represent the Saudi population. We recommend large-scale, multi-center studies on DFSP in the future that will remove possible selection bias and help characterize the behavior of DFSP further in the Saudi population.

In conclusion, dermatofibrosarcoma protuberans is uncommon in the Saudi population, and shows a pattern of disease that is similar to what has been reported worldwide in other populations. Local excision remains the mainstay for the treatment of DFSP, with Imatinib Mesylate at doses of 400 to $800 \mathrm{mg}$ orally daily and radiotherapy considered in certain patients at an advanced stage of the disease.

Acknowledgment. The authors acknowledge the use of Johns Hopkins Aramco Healthcare, Dhahran, Kingdom of Saudi Arabia facilities for the data used in this study. We also thank Dr. Emad Masuadi and Dr. Arulanantham Zechariah Jebakumar for performing the statistical analysis and his support for the results section. We would also like to thank Editage (www.editage.com) for English language editing.

Received 6th June 2021. Accepted Gth September 2021.

From the Department of Dermatology (Al-Dawsari), and from the Department of Pathology (Al Sheikh), Johns Hopkins Aramco Healthcare, Dhahran, Kingdom of Saudi Arabia.

Address correspondence and reprints request to: Dr. Najla A. Al-Dawsari, Department of Dermatology, Johns Hopkins Aramco Healthcare, Dhahran, Kingdom of Saudi Arabia.E-mail: Najla.aldawsari@gmail.com

ORCID ID: https://orcid.org/0000-0003-3133-6116

\section{References}

1. International Agency for Research on Cancer - World Health Organization. Classification of Tumors of Soft Tissue and Bone (Medicine). 5th Edition. Geneva (CH): World Health Organization; 2019. p. 254-262

2. Sirvent N, Maire G, Pedeutour F. Genetics of dermatofibrosarcoma protuberans family of tumors: from ring chromosomes to tyrosine kinase inhibitor treatment. Genes Chromosomes Cancer 2003; 37: 1-19.
3. Kreicher KL, Kurlander DE, Gittleman HR, BarnholtzSloan JS, Bordeaux JS. Incidence and Survival of Primary Dermatofibrosarcoma Protuberans in the United States. Dermatol Surg 2016; 42: S24-S31.

4. Taylor HB, Helwig EB. Dermatofibrosarcoma protuberans: a study of 115 cases. Cancer 1962; 15: 717-725.

5. Parlette LE, Smith CK, Germain LM, Rolfe CA, Skelton H. Accelerated growth of dermatofibrosarcoma protuberans during pregnancy. J Am Acad Dermatol 1999; 41: 778-783.

6. McKee PH, Brenn T, Calonje JE, Granter SR, Lazar AJ, editors. Pathology of the skin with clinical correlations. Missouri (United States): Elsevier; 2005. p. 1729-1735.

7. Patterson JW, editor. Nonmelanocytic Tumors of the Skin. Armed Forces Institute of Pathology (AFIP) Atlas of Tumor Pathology, Series 4. 1st Edition. Amer Registry of Pathology; 2006.

8. Saudi Health Council National Health Information Center. Cancer Incidence Report Saudi Arabia 2015. [UPdayed Nov 2018; Cited 2018 Sept]. Available from URL: https://nhic.gov. sa/eServices/Documents/E\%20SCR\%20final\%206\%20NOV. pdf

9. Al-Dawsari NA, Amra N. Pattern of skin cancer among Saudi patients attending a tertiary care center in Dhahran, Eastern Province of Saudi Arabia. A 20-year retrospective study. Int J Dermatol 2016; 55: 1396-1401.

10. Al-Maghrabi JA, Al-Ghamdi AS, Elhakeem HA. Pattern of skin cancer in Southwestern Saudi Arabia. Saudi Med J 2004; 25: 776-779.

11. Albasri AM, Borhan WM. Histopathological pattern of skin cancer in Western region of Saudi Arabia. An 11 years' experience. Saudi Med J 2018; 39: 994-998.

12. Mufti ST. Pattern of skin cancer among Saudi Patients who attended King AbdulAziz University Hospital between Jan 2000 and Dec 2010. J Dermatol Dermatol Surg 2012; 16 : 13-18.

13. Hafez DM, Alsabaani AA, Al-Atif HM, Ahmadi F, Alqhtani AQ, Alasmar NZ, el at. Patterns of Skin Cancer in a Tertiary Referral Hospital in the Southwestern Region of Saudi Arabia. Med J Cairo Univ 2016; 84: 387-391

14. Alwunais KM, Ahmad S. Pattern of skin cancer at Dammam Medical Complex in Dammam, Saudi Arabia. J Dermatol Dermatol Surg 2016; 1: 51-54. 